\title{
Left to Right Oriented Number Scaling in an Ant
}

\author{
Marie-Claire Cammaerts ${ }^{1} \&$ Roger Cammaerts $^{2}$ \\ ${ }^{1}$ Retired from the Biology of Organisms Department, University of Brussels, Belgium \\ ${ }^{2}$ Retired from the Natural and Agricultural Environmental Studies Department (DEMNA) of the Walloon Region, \\ Belgium \\ Correspondence: Marie-Claire Cammaerts, Independent Researcher, 27, Square du Castel Fleuri, 1170 Bruxelles, \\ Belgium. Tel: 32-2-673-4969. E-mail: mccammaerts@gmail.com
}

Received: August 4, 2019

Accepted: August 19, 2019

Online Published: August 28, 2019

doi:10.5539/ijb.v11n4p67

URL: https://doi.org/10.5539/ijb.v11n4p67

\begin{abstract}
Trained to a smaller number of elements versus a larger one and then tested faced with the larger number and twice the smaller one, one set on the left and the other on the right of the larger number, the ants essentially reacted to the smaller number located on the left of the larger one. Trained to a larger number of elements versus a smaller one and then tested faced with the smaller number and twice the larger one, one set on the left and the other on the right of the smaller number, the ants went preferentially to the larger number located on the right of the smaller one. They similarly reacted when trained to zero versus 2 elements (mostly reacting to the zero element located on the left of the 2 elements), and when trained to 2 elements versus zero element (going essentially to the 2 elements located on the right of the zero element). Thus, the ants responded mostly to the left smaller and the right larger number of elements, and this only when a larger and a smaller respectively number of element was set in the middle. In the absence of the latter, the ants went equally to the left and the right numbers of elements. The ants arrange thus mentally the numbers (amounts) on a scale, a number line, locating the smaller quantities on the left and the larger ones on the right, as do humans and the vertebrates which have already been studied as for this numerosity characteristic. Also, the ants' accuracy of response decreases with increasing numbers of elements.
\end{abstract}

Keywords: Cognition, Conditioning, Myrmica Sabuleti, Number Line, Numerosity

\section{Introduction}

The workers of the ant Myrmica sabuleti Meinert 1861 can distinguish different numbers of elements, can perform what looks to an addition and a subtraction when the results of these operations are concretely presented, the ants responding thus to what they clearly saw during training. These workers have the notion of zero based on visual as well as on olfactory perception, and locate the zero at its correct place, i.e. at the start of an increasing series of elements and at the end of a decreasing series (respectively: Cammaerts, 2008; Cammaerts \& Cammaerts, 2019c, d, f, 2019 a, b, e).

Do ants have any number representation allowing them having the here above enumerated numerosity capabilities, or do they have these capabilities without possessing any mental representation of numbers or quantities? Such a question has been answered for humans. The latter possess a number representation (Dehaene, Dehaene-Lamberts, \& Cohen, 1998), a cerebral property detailed in Dehaene (2011). The question has been seldom addressed for animals, maybe due to the difficulty of experimenting on the subject, and until now was addressed only in vertebrates. Agrillo (2012) has reported some number representation in guppies (fishes). Rugani, Vallortigara, Priftis and Regolin (2015) went a step further. Thanks to experiments, they showed that very young chicks situate the smaller amounts on the left and the larger ones on the right. While studying the potential ability of pigeons in making subtractions, Brannon, Wusthoff, Gallistel and Gibbon (2001) accidently discovered that these birds may have an innate number representation, locating the numbers on a scale. It has also been revealed that monkeys mentally and spontaneously arrange the numbers on a scale (Brannon \& Herbert, 1988; Hauser, Carey, \& Hauser, 2000).

Until now, the problem of number representation appears to have not been examined in any invertebrates. Thanks to several studies of the numerosity abilities of $M$. sabuleti workers (see references above), we have acquired a rather good knowledge these ants' performance as for this topic, but we still ignore if they possess any number representation. We thus intended to fill this gap by experimenting on six ant colonies. We trained the ants to a given number of elements set near their food, and at the same time, to another number of elements, either larger 
or smaller, set far from the food. Then, we tested these ants faced with twice the number of elements they saw near their food during training, these numbers being located on the left and on the right of the number of elements they saw far from the food during training. During these tests, if the ants have no number representation, they would respond equally to the numbers of elements set near the food during training and located during the tests on the left and on the right of the number set far from the food. If they have a number representation, they would prefer either the left or the right of these two numbers to which they were conditioned. Our experiments included also the zero as a number of elements. After having explained our methods and reported our results, we discuss the latter and somewhat extend our finding.

\section{Material and Methods}

\subsection{Collection and Maintenance of Ants}

The experiments were performed on six colonies of M. sabuleti (labeled A to F) collected in abandoned quarries, in June 2018 at Marchin (Condroz, Belgium), and in September 2018 in the Aise valley (Ardenne, Belgium). Each colony contained about 500 workers, brood and a queen. They were maintained in the laboratory in two to four glass tubes half filled with water, a cotton plug separating the ants from the water. The nest tubes of each colony were set in a tray $(34 \mathrm{~cm} \times 23 \mathrm{~cm} \times 4 \mathrm{~cm})$ which served as foraging area. In this area, pieces of Tenebrio molitor larvae (Linnaeus, 1758) were provided three times per week and cotton plugged tubes filled with sugar water were permanently set. The ambient temperature was $c a 20^{\circ} \mathrm{C}$, the humidity $80 \%$, the lighting 330lux while working on ants, and the electromagnetism $2 \mu \mathrm{Wm}^{2}$. All these environmental conditions were optimum for the species.

\subsection{Cues Presented to the Ants}

Three experiments, labeled I, II, III, were performed using different numbers of green, yellow and violet rectangles respectively. We used three different colors, one for each experiment, to avoid any memorization for one experiment to the following one since the ant colonies used for these three experiments were the same. Note that the ant M. sabuleti distinguishes very well the colors, even under low light intensity (Cammaerts, 2007; Cammaerts M. C., \& Cammaerts D., 2009). The colored rectangles were drawn using software Power Point ${ }^{\circledR}$, printed and tied each one on a stand (vertical part: $2.5 \mathrm{~cm} \times 2.5 \mathrm{~cm}$ ) made of strong white paper. These stands were maintained vertically thanks to their folded base $[2 \times(0.5 \mathrm{~cm} \times 1.25 \mathrm{~cm})]$ (Figure 1, upper part).

Experiments I and II were made of three parts, experiment III of two parts (Figure I). Each experimental part was made of training and testing sessions, each session having its own new stands bearing the colored rectangles (Figure 2).

During experiment I, the ants of colonies A and B were trained to 1 rectangle set near their food versus 3 rectangles set far from the food. They were tested faced with 3 rectangles set in the middle, with 1 rectangle set on the left, and with 1 rectangle set on the right of the 3 rectangles. In the same way, the ants of colonies $C$ and $D$ were trained to 2 rectangles set near their food versus 4 rectangles set far from the food. They were tested faced with 4 rectangles set in the middle, with 2 rectangles set on the left, and with 2 rectangles set on the right of the 4 rectangles. Similarly, the ants of colonies $\mathrm{E}$ and $\mathrm{F}$ were trained to 3 rectangles set near their food versus 5 rectangles set far from the food. They were tested faced with 5 rectangles set in the middle, with 3 rectangles set on the left, and with 3 rectangles set on the right of the 5 rectangles. Thus, during experiment I, the ants were trained to a small number of rectangles versus a larger number, and tested in front of the small number located on the left and on the right of the larger number.

During experiment II, the ants of colonies A and B were trained to 4 rectangles set near their food versus 2 rectangles set far from the food. They were tested faced with 2 rectangles set in the middle and with twice 4 rectangles, one on the left and the other on the right of the 2 rectangles. In the same way, the ants of colonies $\mathrm{C}$ and D were trained to 5 rectangles set near their food versus 3 rectangles set far from the food. They were tested faced with 3 rectangles set in the middle and with twice 5 rectangles, one on the left and the other on the right of the 3 rectangles. Similarly, the ants of colonies E and F were trained to 3 rectangles set near their food versus 1 rectangle set far from the food. They were tested faced with 1 rectangle set in the middle and with twice 3 rectangles, one on the left and the other on the right of the 1 rectangle. Thus, during experiment II, the ants were trained to a large number of rectangles versus a smaller number, and tested in front of the large number located on the left and on the right of the smaller number. On the basis of the obtained results, a complementary experimental observation was decided to be done, six hours later, on the same still trained ants, testing them faced with only the number of elements set on the right and the left, and no longer with the number previously set in the middle (this is not illustrated in Figure 1). 
During experiment III, the ants of colonies A and B were trained to 0 rectangle set near their food versus 2 rectangles set far from the food. They were tested faced with 2 rectangles set in the middle and twice 0 rectangle, one on the left and the other on the right of the 2 rectangles. Inversely, the ants of colonies $\mathrm{C}$ and $\mathrm{D}$ were trained to 2 rectangles set near their food versus 0 rectangle set far from the food. They were tested faced with 0 rectangle set in the middle and with twice 2 rectangles, one on the left and the other on the right of the 0 rectangle. The first part of experiment III corresponded to experiment I; the second part of experiment III corresponded to experiment II; each time, 0 rectangle was used as the small number of rectangles. Such as for experiment II, on the basis of the obtained results, a complementary experimental observation were decided to be done, six hours later, on the same still trained ants, testing them faced with only the number located on the right and on the left, and no longer with the number previously set in the middle (this is not illustrated in Figure 1).

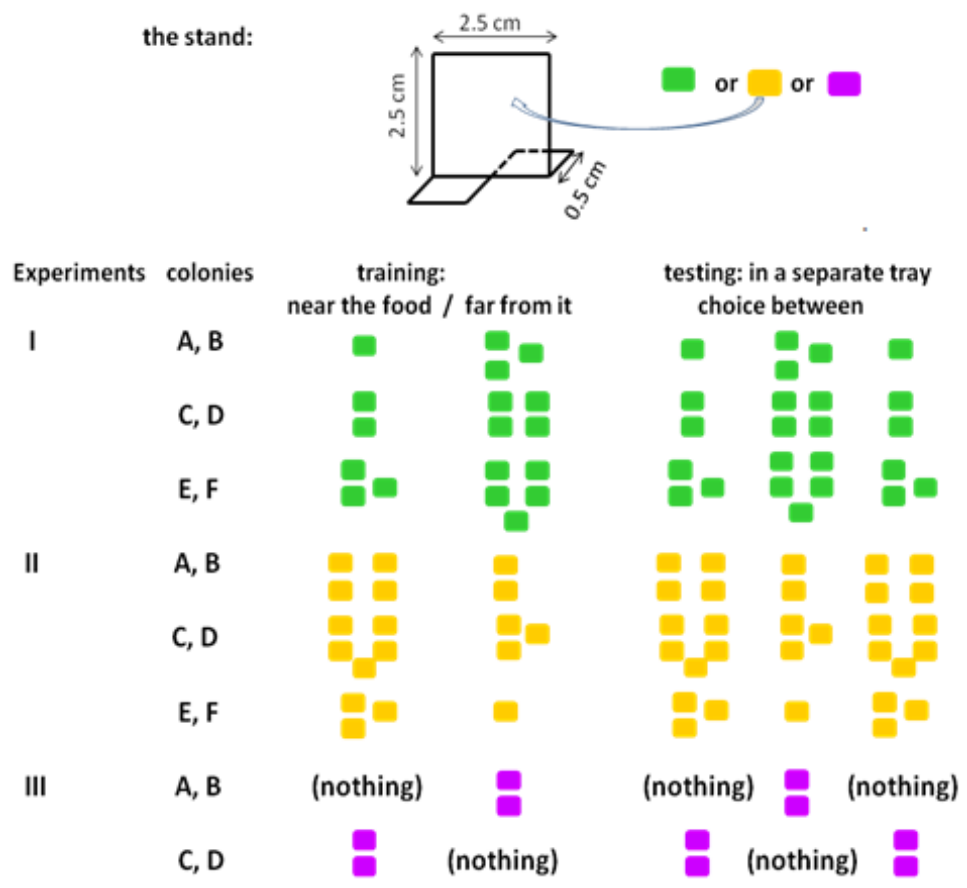

Figure 1. Schema of the different amounts of rectangles presented to the ants during their training and testing

During training: for experiment I, the smaller amount was near the food; for experiment II, the larger amount was near the food; for experiment III, the amounts were either zero (0) near the food or 2 near the food. During testing: the amount set near the food during training was presented twice, i.e. on the left and on the right of the amount set far from the food during training. Results are given in Tables 1, 2 and 3; photos are shown in Figures 3, 4, and 5.

\subsection{Experimental Design and Protocol}

The ants' training was performed on their foraging area: a stand bearing a given number of rectangles was set aside the food; another stand bearing another number of rectangles was set far from the food. During training, the ants could easily see the two stands, and memorize which one was aside their food (Figure 2, lower part). During each part of each experiment, the ants of the two used colonies were trained during two days. At each training day, the ants of the two colonies being in the surrounding of the two presented stands were counted six times over each day, and the two mean values were established (Tables 1,2,3, upper part). The total mean was also calculated and is given in the text only.

The ants were then tested in a separate tray $(30 \mathrm{~cm} \times 15 \mathrm{~cm} \times 4 \mathrm{~cm})$, one for each used colony. The borders of these trays were slightly covered with talc to prevent the ants escaping, and in them, three stands (or two during the complementary observations) bearing the numbers of rectangles defined here above (see Cues presented to the ants) were set at about $8 \mathrm{~cm}$ from one another. To make a test, 25 ants of each two used colonies were transferred into their tray devoted to testing, and the ants present at a distance of $0-2 \mathrm{~cm}$ from each of the stands were counted 20 times over 10 experimental minutes. After each test, on each colony, the 20 ants were replaced in their nest. The means of these counts were established (Tables 1, 2, 3, lower part). The total numbers and the proportions of ants having responded to each stand were also calculated and are given only in the text, not in a table. 


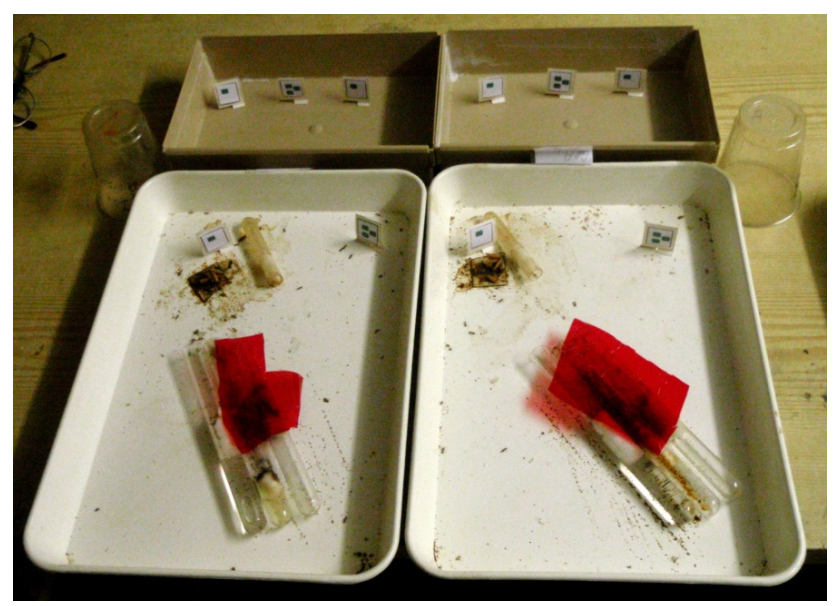

Figure 2. Experimental design

The ants were trained to a small or a large amount of rectangles set on a stand near their food versus respectively a large or a small amount of rectangles set far from the food (lower part of the figure). They were then tested in a separate tray in front of the small (or the large) amount, set on the left as well as on the right of a stand with the large (or the small) amount (upper part of the figure). The different used amounts are schematized in Figure 1, and photos of the experiments are given in Figures 3, 4, and 5.

\subsection{Statistical Analysis}

The data recorded during training did not require any statistical analysis.

Those recorded during testing were statistically analyzed as follows. For each test, the 20 numbers of ants of the two used colonies having responded to each presented stands were chronologically arranged and added by four over the experimental time. The five sums (given only in the text) obtained for each stand were compared to one another using the non-parametric test of Wilcoxon (Siegel \& Castellan, 1989).

\section{Results}

\subsection{Experiment $I$}

Numerical results are given in Table 1 and views of the experiment can be seen in Figure 3.

When trained to 1 green rectangle set near their food and to 3 green rectangles set far from the food, the ants of colonies A and B were rather numerous at any time all around near these two cues. They were meanly 10 in doing so. After two training days, the tested ants of colonies A and B reacted similarly. They went essentially towards the 1 rectangle located on their left $(37+45=82$ ants did so, i.e. $63.6 \%)$. They went also towards the 1 rectangle located on their right $(15+15=30$ ants did so, i.e. $23.3 \%)$. Less ants were counted near the 3 rectangles located in the middle $(10+7=17$ ants were counted there, i.e. 13.2\%). Summed by four over the experimental time, the numbers of ants seen near the 1 rectangle set on the left equaled 17,16,17, 17, 15, those of ants seen near 3 rectangles equaled $3,7,3,0,4$, and those of ants seen near the 1 rectangle set on the right equaled 5, 8, 7, 6, 4. The most similar series of these three series of numbers are that relative to the 1 rectangle set on the left and that relative to the 1 rectangle set on the right. These two more similar series statistically differed $(\mathrm{N}=5, \mathrm{~T}=15, \mathrm{P}=0.031)$. The ants reacted thus more to the 1 rectangle located on their left than to that located on their right, and of course more to these 1 rectangles than to 3 rectangles.

Trained to 2 green rectangles set near their food and to 4 ones set far from it, many ants of colonies C and D foraged at any time all round these two cues. They were meanly 9.2 ants in doing so, and could thus sufficiently see and memorize the presented cues. When tested after two days, the ants of colonies $\mathrm{C}$ and $\mathrm{D}$ had a similar behavior: they moved preferentially to the stand bearing 2 rectangles located on the left of the stand bearing 4 rectangles, and at a lower extent, to the stand bearing 2 rectangles located on the right of that bearing 4 rectangles. In total, 145 ants, i.e. $73.2 \%$, were counted near the 2 rectangles located on the left, 20 ants, i.e. $10.1 \%$, near the 4 rectangles located in the middle, and 33 ants, i.e. $16.7 \%$, near the 2 rectangles located on the right. Summed by four over the experimental time, the numbers of ants seen in front of the 2 rectangles set on the left equaled 36, 32, 29, 21, 27, those of ants seen in front of the 4 rectangles equaled 12, 4, 1, 1, 2, and those of ants seen in front of the 2 rectangles set on the right equaled $7,6,6,6,8$. The smallest difference occurred between the series of five numbers counted in front of the 2 rectangles set on the left and the series of five numbers counted in front of the 2 rectangles set on the right. This 
smallest difference was already statistically significant $(\mathrm{N}=5, \mathrm{~T}=15, \mathrm{P}=0.031)$. Thus, the ants have more often chosen the 2 rectangles set on the left of the 4 ones than the 2 rectangles set on the right of the 4 ones.

During their training to 3 green rectangles set near the food and 5 ones set far from it, the ants of colonies $\mathrm{E}$ and $\mathrm{F}$ were numerous at any time in foraging in the vicinity of these two cues. Meanly 10.9 ants were counted there, enough for allowing them seeing and memorizing the presented cues. When tested, the ants of the two colonies similarly reacted: most of them moved to the 3 rectangles located on the left, less ones moved to the 3 rectangles located on the right, and only few ones went to the 5 rectangles located in the middle. In total, 137 ants, i.e. $73.3 \%$, were seen in front of the 3 green rectangles located on the left, 17 ants, i.e. $9.1 \%$, in front of the 5 green rectangles, and 33 ants, i.e. $17.1 \%$, in front of the 3 rectangles located on the right. Summed by four over the experimental time, the numbers of ants counted near the 3 rectangles set on the left equaled 34, 41,21,20,21, of ants counted near the 5 rectangles equaled $7,4,2,1,3$, and of ants counted near the 3 rectangles set on the right equaled 7,6 , $5,7,8$. The less different series among these three series of numbers were those relative to the 3 rectangles set on the left and to the 3 rectangles set on the right, and these series nevertheless statistically differed $(\mathrm{N}=5, \mathrm{~T}=15, \mathrm{P}$ $=0.031$ ). Thus, the ants preferentially reacted to the 3 rectangles located on the left of the 5 rectangles, reacted at a lower extend to the 3 rectangles set on the right of the 5 rectangles, and reacted poorly to the 5 rectangles.

The results of the three parts of experiment I were in agreement with one another. Trained to 1, 2, 3 rectangles set near their food versus 3, 4, 5 rectangles set far from the food, the tested ants reacted more to the rectangles presented on the left of the larger number of rectangles presented in the middle than to the rectangles presented on the right of this larger number of rectangles. The ants reacted thus the best to a smaller amount located on the left of a larger amount. Did they behave similarly when the large amount was set near the food and the small amount set far from the food during training? Experiment II tried to answer this question.

Table 1. Results of experiment I done to examine if ants locate the smaller amounts on the left of larger ones

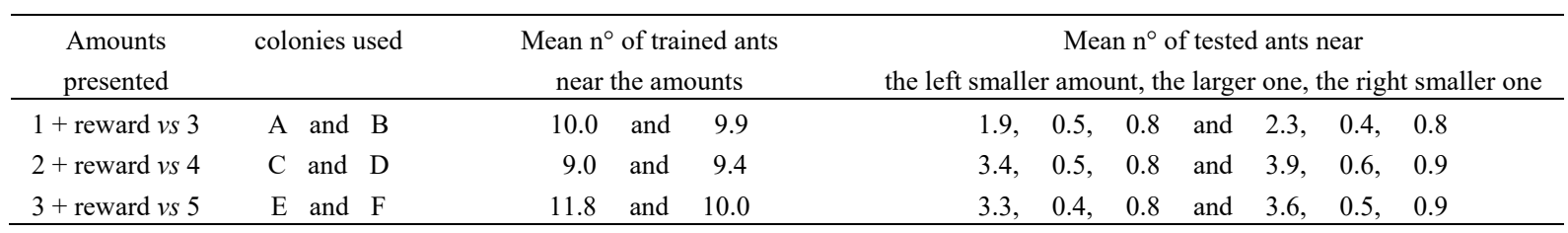

Details and statistics are given in the text; photos are shown in Figure 3. Briefly, when tested, the ants were more numerous near the smaller amount set on the left of the larger amount than near the smaller amount set on the right.

\subsection{Experiment II}

Numerical results can be found in Table 2 and some photos of the experiment are presented in Figure 4.

While being trained to 4 yellow rectangles set near their food $v s 2$ yellow rectangles set far from the food, the ants of colonies A and B were rather numerous at any time all around these two cues. In fact, they were meanly 7.6 in doing so. When tested in front of twice 4 rectangles, one on the left and the other on the right of the stand with 2 rectangles, the ants of colonies A and B similarly reacted: they essentially went towards the 4 rectangles located on the right of the 2 rectangles. In total, 90 ants, i.e. $69.8 \%$, were counted in front of the 4 rectangles located on then right, 18 ants, i.e. $14 \%$, in front of the 2 rectangles, and 21 ants, i.e. $16.3 \%$ in front of the 4 rectangles located on the left. Summed by four over the experimental time, the numbers of ants seen near the 4 rectangles set on the right equaled 17, 16, 24, 22, 11, near the 2 rectangles equaled $6,1,6,3,2$, and near the 4 rectangles set on the left equaled $2,1,7,10,1$. The first and the last of these three series of five numbers statistically differed $(\mathrm{N}=5, \mathrm{~T}=$ $15, \mathrm{P}=0.031$ ). Consequently, the ants mostly reacted to the 4 rectangles located on the right and far less to the 4 rectangles located on the left. Six hours later, a complementary experimental observation was done: the ants were again tested, however without presenting them the stand with the 2 rectangles previously set in the middle, thus presenting them only twice the 4 rectangles. The ants of the two colonies equally reacted to the two stands bearing 4 rectangles. In total, 60 ants, i.e. $50.4 \%$, were counted in front of the 4 rectangles located on the left, and 59 ants, i.e. $49.6 \%$, were counted in front of the 4 rectangles located on the right. Summed by four over the experimental time, the numbers of ants seen near the 4 rectangles set on the left equaled 20,10, 9, 8, 13, and of ants seen near the 4 rectangles set on the right equaled $15,14,10,9,11$. These two series of five numbers did not statistically differ $(\mathrm{N}=5, \mathrm{~T}=-8,+7, \mathrm{P}=0.50)$. Consequently, the ants went to the 4 rectangles located on the right only in presence of the 2 rectangles between the two 4 rectangles. 
During their training to 5 rectangles set near the food $v s 3$ rectangles, the ants of colonies $\mathrm{C}$ and $\mathrm{D}$ were numerous enough all around these two cues for perceiving and memorizing them. They were meanly 8.9 on this area. When tested, the ants of the two colonies similarly went essentially to the stand bearing 5 yellow rectangles located on the right of that bearing 3 yellow rectangles. In total, 163 ants, i.e. $78.4 \%$, were counted near the 5 rectangles located on the right, 21 ants, i.e. $10.1 \%$, were counted near the 3 rectangles located in the middle, and 24 ants, i.e. $11.5 \%$, near the 5 rectangles located on the left. Summed by four over the experimental time, the numbers of ants seen in front of the right 5 rectangles equaled 36, 40,32, 28, 27, those of ants seen in front of the 3 rectangles equaled 4, 1, 6, 7, 3, and those of ants seen in front of the left 5 rectangles equaled $3,3,6,6,6$. The first and the last of these three series of five numbers statistically differed $(\mathrm{N}=5, \mathrm{~T}=15, \mathrm{P}=0.031)$. The ants have thus reacted more to the 5 rectangles set on the right of the 3 rectangles than to the 5 rectangles set on the left of these 3 rectangles. Six hours later, a complementary experimental observation was done, the ants being still trained. The same ants were tested in front of only the two stands bearing each one 5 yellow rectangles, one staying on the right, the other on the left in the tray. This time, the ants of the two colonies went equally towards each of the two stands. In total, 113 ants, i.e. 50.9\%, were counted in front of the left stand and 109 ants, i.e. $49.1 \%$, were counted in front of the right stand. Summed by four over the experimental time, the numbers of ants seen near the 5 rectangles set on the left equaled 15, 20,24, 25, 29, and those of ants seen near the 5 rectangles set on the right equaled 15, 20, $27,20,27$. These two series of five numbers did not statistically differ $(\mathrm{N}=3, \mathrm{NS})$. The ants went thus essentially to the 5 rectangles set on the right and less to those set on the left only when these cues were presented with the stand bearing 3 rectangles set between them.

When trained to 3 yellow rectangles set near their food and to 1 such rectangle set far from the food, the ants of colonies $\mathrm{E}$ and $\mathrm{F}$ were rather numerous in foraging all around these two cues. They were meanly 10.1 in doing so at any time. When tested, the ants of the two colonies behaved similarly: they went essentially to the stand bearing 3 yellow rectangles located on the right of that bearing 1 rectangle. In total, 115 ants, i.e. $75.7 \%$, were counted near the 3 rectangles set on the right, 15 ants, i.e. $9.9 \%$, were counted near the 1 rectangle located in the middle, and 22 ants, i.e. $14.5 \%$, were counted near the 3 rectangles set on the left. Summed by four over the experimental time, the numbers of ants seen in front on the 3 rectangles located on the right equaled 25, 27, 21,23, 19, of ants seen in front of the 1 rectangle set in the middle equaled 4, 4, 3, 2, 2, and of ants seen in front of the 3 rectangles located at the left equaled $5,7,4,2,4$. The first and the third of these three series of five numbers statistically differed $(\mathrm{N}=5, \mathrm{~T}=15, \mathrm{P}=0.031)$. The ants have thus mostly reacted to the 3 rectangles located on the right of the 1 rectangle. Six hours later, while the ants' training went on, a complementary experimental observation was done. The same ants were tested in front of only the right and the left 3 rectangles. This time, the ants of the two colonies went about equally to the two differently located 3 rectangles. In total, 69 ants, i.e. $46.3 \%$, were counted in front of the 3 rectangles located on the right, and 80 ants, i.e. $53.7 \%$, were counted in front of the 3 rectangles located on the left. They went thus slightly more to the left rectangles, contrary to the previous observation. However, summed by four over the experimental time, the numbers of ants seen near the 3 rectangles set on the right equaled 20,12,15,14, 8, and of ants seen near the left 3 rectangles equaled $19,20,19,12,10$. These two series of five numbers did not statistically differ $(\mathrm{N}=5, \mathrm{~T}=-3.5$, $+11.5, \mathrm{P}=0.266, \mathrm{NS})$. The ants went thus mostly to the 3 rectangles located on the right only when this cue was presented twice at both sides of the stand with 1 rectangle.

The three parts of this experiment II leaded to agreeing results. Each time, the ants reacted essentially to the larger amount located on the right of a smaller amount, and did not go preferentially to that right located amount in the absence of the smaller one.

Table 2. Results of experiment II done to examine if ants locate the larger amounts on the right of smaller ones

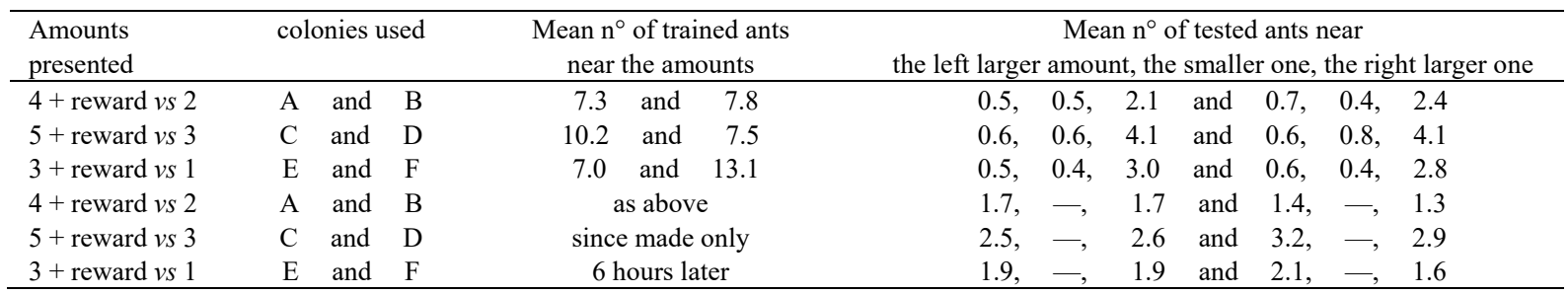

Details and statistics are given in the text; photos are shown in Figure 4. Upper part of the table: the tested ants were more numerous near the larger amount set on the right of the smaller amount than near the larger amount set on the left. Lower part of the table: the ants were equally numerous near two identical amounts, located on the right and on the left, when no other amount was set in the middle. The ants preferred thus the larger amount set on the right only in the presence of a smaller amount between the two larger amounts. 


\subsection{Experiment III}

Numerical results are presented Table 3 and some photos of the experiment in Figure 5.

When trained to 0 element set near their food versus 2 violet rectangles set far from the food, the ants of colonies $\mathrm{A}$ and $\mathrm{B}$ were numerous enough at any time all around these two cues for seeing and memorizing them, being meanly 9.3 to do so. When tested, the ants of colonies A and B similarly went essentially to the stand bearing 0 violet rectangle located on the left of that bearing 2 rectangles. In total, 112 ants, i.e. $75.7 \%$, were counted in front of the 0 rectangle located on the left, 20 ants, i.e. $13.5 \%$, were counted in front of the 2 rectangles, and 16 ants, i.e. $10.8 \%$, were counted in front of the 0 rectangle located on the right. Summed by four over the experimental time, the numbers of ants seen near the 0 rectangle set on the left equaled 16,24,24, 27, 21, the numbers of ants seen near the 2 rectangles equaled $3,4,5,6,2$, and those of ants seen near the 0 rectangle set on the right equaled 0,3 , $4,6,3$. The first and the third of these three series of numbers statistically differed $(\mathrm{N}=5, \mathrm{~T}=15, \mathrm{P}=0.031)$. The ants went thus preferentially to the 0 rectangle located on the left of the 2 rectangles. During a complementary experimental observation, when tested in front of only the two 0 rectangles, i.e. without the stand bearing 2 rectangles and set in the middle, the still trained ants of the two colonies went nearly identically to the two stands bearing 0 rectangle. Indeed, 103 ants, i.e. 50.7\%, were counted near the left 0 rectangle, and 101 ants, $49.3 \%$, were counted near the right 0 rectangle. Summed by four over the experimental time, the numbers of ants seen in front the left 0 rectangle equaled 17, 18,27,21,20, and those of ants seen in front of the right 0 rectangle equaled 18 , $17,25,21,20$. These two series of five numbers did not statistically differ $(\mathrm{N}=3$, NS). The ants went thus preferentially to the stand with 0 rectangle located on the left only when there was a stand with 2 rectangles set in the middle, between the two stands with 0 rectangle.

When trained to a stand with 2 violet rectangles set near their food versus a stand bearing 0 rectangle set far from the food, the ants of colonies $\mathrm{C}$ and $\mathrm{D}$ were rather numerous in foraging at any time in the vicinity of these two cues, being meanly 9 in doing so. After two training days, the tested ants of colonies $\mathrm{C}$ and $\mathrm{D}$ moved mostly towards the 2 violet rectangles located on the right of the stand with 0 rectangle. In total, 185 ants, i.e. $75.8 \%$, were counted in front of the stand with 2 rectangles set on the right, 32 ants, i.e. $13.1 \%$, were counted in front of the 0 rectangle set in the middle, and 27 ants, $11.1 \%$, were counted in front of the 2 rectangles set on the left. Summed by four over the experimental time, the numbers of ants seen near the 2 right rectangles equaled 37, 41,37, 32, 38, seen near the 0 rectangle equaled $9,8,3,8,4$, and seen near the left 2 rectangles equaled $6,4,5,4,8$. The first and third of these three series of five numbers statistically differed $(\mathrm{N}=5, \mathrm{~T}=15, \mathrm{P}=0.031)$. The ants went thus preferentially to the stand with 2 rectangles located on the right of the stand with 0 rectangle. When the same still trained ants were tested, in the course of a complementary experimental observation, faced with two 2 rectangles, one set on the left, the other on the right, without the stand with 0 rectangle in the middle, they went nearly equally towards the two 2 rectangles. Indeed, 96 ants, i.e. $49.7 \%$, were counted in front of the right stand with 2 rectangles, and 97 ants, i.e. $50.3 \%$, were counted in front of the left stand with 2 rectangles. Summed by four over the experimental time, the numbers of ants having approached the right located 2 rectangles equaled 21, 17, 23, 21, 14, and those of ants having approached the left located 2 rectangles equaled 20,22, 18,20,17. These two series of five numbers did not statistically differ $(\mathrm{N}=$ $5, \mathrm{~T}=+7.5,-7.5, \mathrm{P}>0.50$ ). The ants moved thus preferentially to the stand bearing 2 rectangles located on the right only in the presence of a stand bearing 0 rectangle set in the middle.

The results of the first part of experiment III were in agreement with those of experiment I, and the results of the second part of experiment III were in agreement with those of experiment II.

Table 3. Results of experiment III made for examining if ants locate the zero on the left of 2 elements, and 2 elements on the right of the zero

\begin{tabular}{|c|c|c|c|c|c|c|c|c|}
\hline \multirow{2}{*}{$\begin{array}{l}\text { Cues presented } \\
0+\text { reward } v s 2\end{array}$} & \multicolumn{2}{|c|}{ colonies used } & $\begin{array}{c}\text { Mean } n^{\circ} \text { of trained ants } \\
\text { near the cues }\end{array}$ & \multicolumn{5}{|c|}{$\begin{array}{l}\text { Mean } n^{\circ} \text { of tested ants near } \\
\text { the left correct cue, the other cue, the right correct cue }\end{array}$} \\
\hline & and & $\mathrm{B}$ & 9.5 and 9.1 & $3.3, \quad 0.6$ & 0.4 & and & $2.4, \quad 0.5$ & 0.4 \\
\hline $2+$ reward $v s 0$ & $\mathrm{C}$ and & $\mathrm{D}$ & 9.9 and 8.0 & 0.8, 0.9, & 4.9 & and & $0.6, \quad 0.8$ & 4.4 \\
\hline $0+$ reward $v s 2$ & A and & B & as above since & 2.4, 一, & 2.3 & and & $2.8,-$ & 2.8 \\
\hline $2+$ reward $v s 0$ & $\mathrm{C}$ and & $\mathrm{D}$ & made 6 hours later & 2.0, - & 2.2 & and & 2.9 & 2.6 \\
\hline
\end{tabular}

Details and statistics are given in the text and photos are shown in Figure 5. Briefly, the ants went essentially to the 0 element located on the left of 2 elements, and to the 2 elements located on the right of 0 element (upper part of the table), and a complementary experimental observation (lower part of the table) showed that the ants went equally to the left and the right 0 or 2 elements in the absence of respectively the 2 or 0 elements in the middle. All this is in agreement with the results of experiments I and II given in Tables 1 and 2. 
training testing

Colonies A and $\mathrm{B}, 1$ vs 3
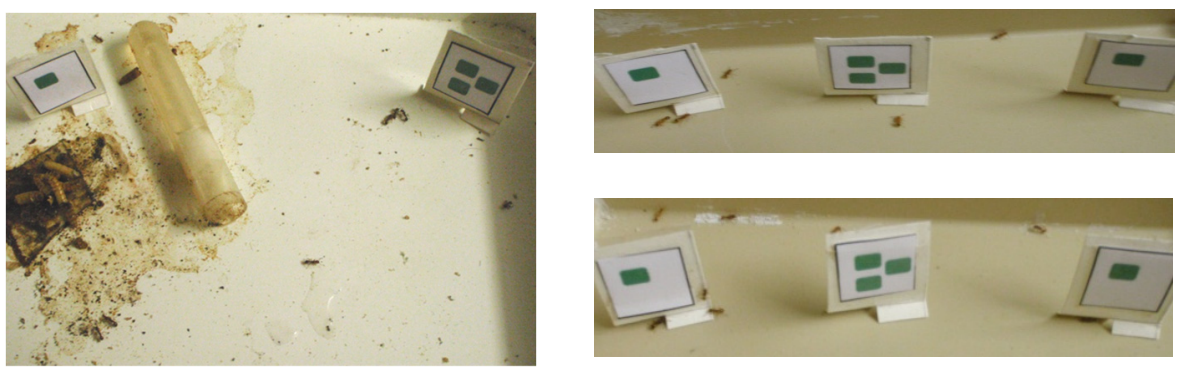

Colonies C and D, 2 vs 4
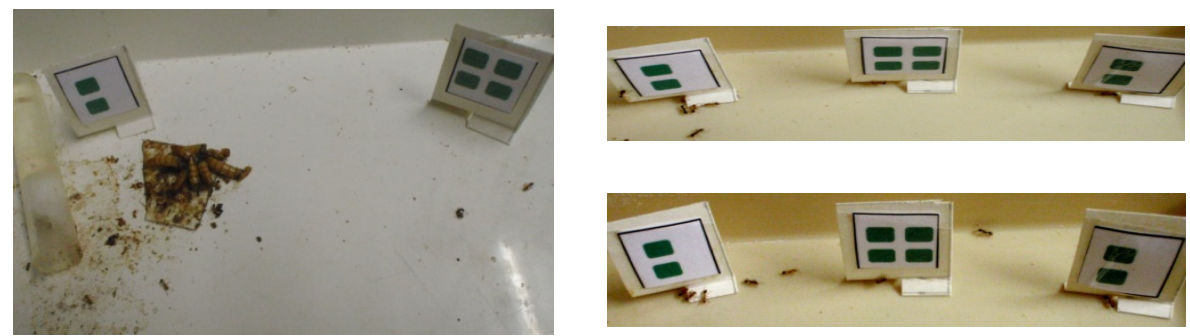

Colonies E and F, 3 vs 5
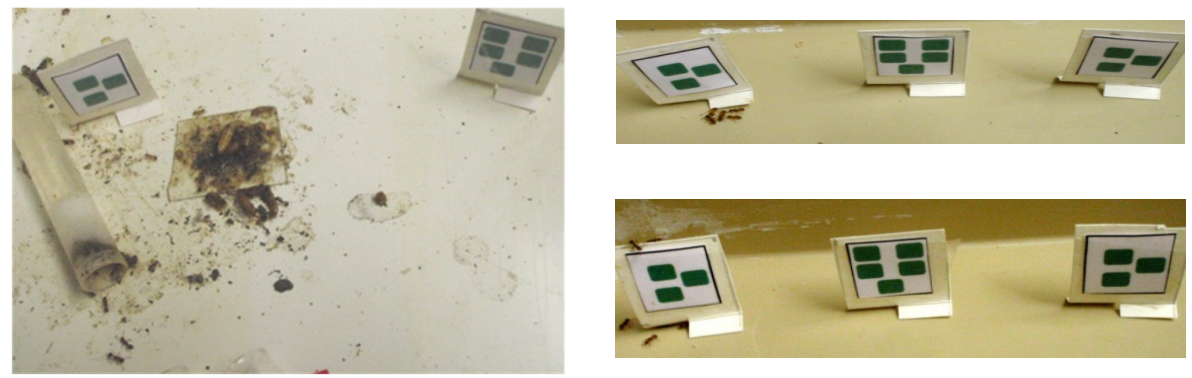

Figure 3. Some views of experiment I

Details are given in the text and results in Table 1. Briefly, after having been trained to 1, 2 or 3 green rectangles set near their food, versus to respectively 3, 4, or 5 green rectangles set far from the food (left part of the figure), the tested ants went essentially to the 1,2, or 3 rectangles located on the left of the 3 , 4, or 5 green rectangles. 


$$
\text { training testing }
$$

Colonies A and B, 4 vs 2


Colonies $\mathrm{C}$ and $\mathrm{D}, 5$ vs 3


Colonies E and F, 3 vs 1
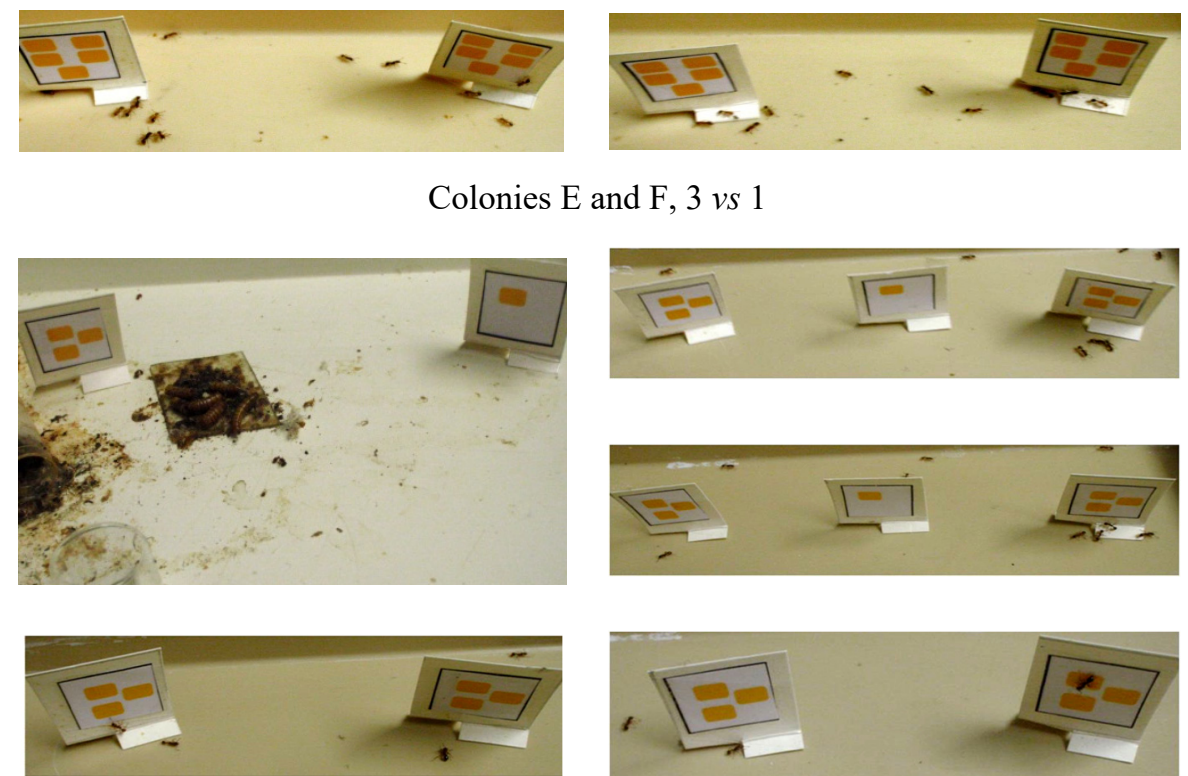

Figure 4. Some views of experiment II

Details are given in the text, schemas of the amounts of presented rectangles in Figure 1, and numerical results in Table 2. Briefly, after having been trained to a larger amount of yellow rectangles set near the food versus a smaller one set far from the food (left part of the figure), when tested faced with twice the larger amount, one set on the left and the other on the right of the smaller amount, the ants went essentially to the larger amount located on the right of the smaller one (right part of the figure). The two lower photos for each three parts of the experiment show that the ants did so only in the presence of the smaller amount presented between the two larger amounts. 
Colonies A and B, 0 vs 2
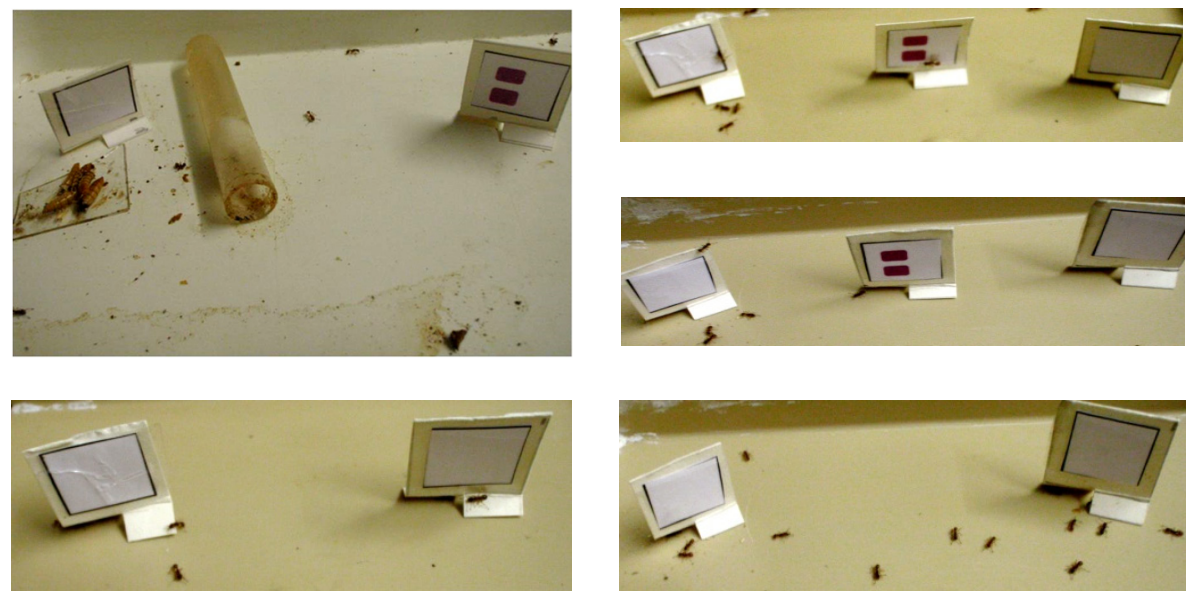

Colonies C and D, 2 vs 0
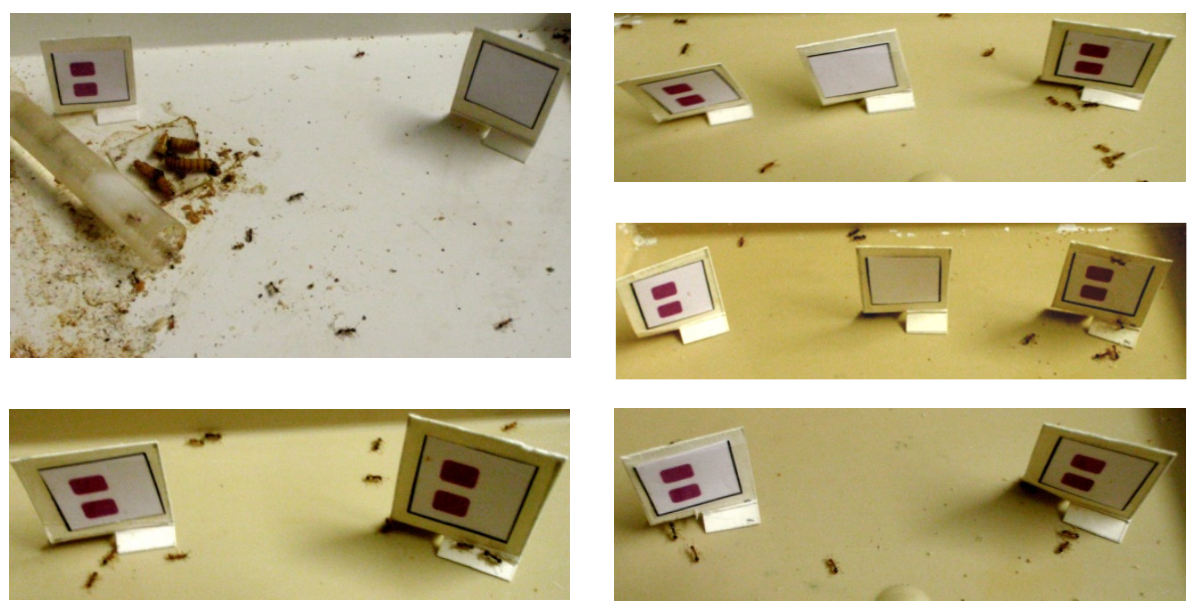

Figure 5. Some views of experiment III

Details are given in the text, schemas of the presented rectangles in Figure 1, and numerical results in Table 3 . The ants, trained to a stand bearing 0 rectangle set near the food versus a stand with 2 violet rectangles set far from the food, when being tested, went essentially to the 0 rectangle located on the left of the 2 rectangles, and equally to the left and the right located 0 rectangle in the absence of the 2 rectangles (upper part of the figure). Trained to 2 rectangles set near the food versus to 0 rectangle set far from the food, the tested ants went essentially to the 2 rectangles located on the right of the 0 rectangle, and equally to the left and the right stands with 2 rectangles in the absence of the 0 rectangle (lower part of the figure). This is in agreement with what occurred during experiments I and II.

\subsection{Comparison of Experiments I, II, III}

Experiment I and the first part of experiment III revealed that the ants trained to a small amount, and tested faced with such a small amount presented on both side of a larger amount went essentially to the small amount set on the left of the larger amount. The ants did so with a score of about $70 \%$ (scores obtained during the three parts of experiment I: $63.6 \%, 73.2 \%, 73.3 \%$; score obtained during the first part of experiment III: $75.7 \%$; mean of the four scores $=71.5 \%$ ). Experiment II and the second part of experiment III showed that the ants trained to a large amount, and tested faced with such a large amount on the left and on the right of a smaller amount, went essentially to the large amount set on the right. The ants did so with a score slightly higher than $70 \%$ (scores obtained during the three parts of experiment II: $69.8 \%, 78.4 \%, 75.7 \%$; score obtained during the second part of experiment III: $75.8 \%$; mean of the four scores $=74.0 \%$ ). Complementary experimental observations done during experiments II and III also pointed out that the ants presented a 'polarized' choice of the numbers (amounts) set on the left or on 
the right, only if a larger or a smaller number was set between them. The ants appeared thus to arrange amounts on a scale, locating the smaller amounts on the left and the larger ones on the right.

What kind of arrangement may exist on the ants' left to right oriented number line (i.e. from the smaller to the larger amounts)? For example, is it a linear or a logarithmic arrangement? For tempting answering this question, we used the results of a previous work dealing with the subtracting-like ability of M. sabuleti ants (Cammaerts \& Cammaerts, 2019f). In this work, we made two similar experiments for assessing the ants' responses to the subtractions 2 minus 1, 3-1, 4-1, and 5-1. They led to identical results. Here we use the mean of these results, i.e. the ants' mean scores obtained for the four presented subtractions. For 2-1, 3-1, 4-1, and 5-1, these mean scores were $94.0 \%, 90.2 \%, 85.3 \%$, and $82.2 \%$ respectively. The ants' response decreased thus when the sighted amount increased. The relation between the ants' responses and the sighted amounts reflects the kind of arrangement of the amounts on the number line. For approaching the nature of this relation, we arbitrarily replaced 2-1, 3-1, 4-1, and $5-1$ by $1.5,2.5,3.5$, and 4.5 , the latter numbers approaching the best what the ants could see. We observed that the ants' responses appeared to linearly decrease with these arbitrarily chosen values (Figure 6). The ants' responses appeared to less well linearly decrease in relation with the decimal logarithm of these arbitrary chosen values (i.e. $0.17,0.39,0.54$ and 0.65 ) (Figure 6). The ants may thus rather linearly arrange the numbers (the amounts) on a number line. However, further investigations are needed to define the ants' arrangement of the numbers on the number line.

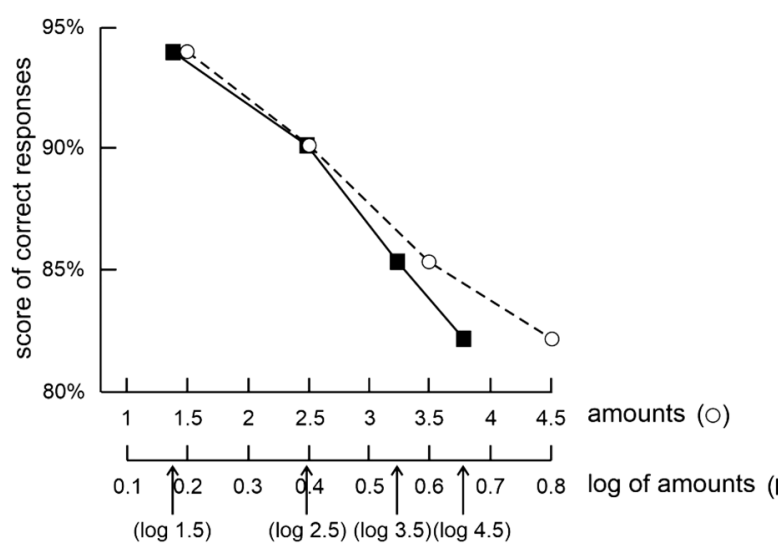

Figure 6. Ants' response to four different numbers of elements

This graph suggests that, in the ant M. sabuleti, the arrangement of numbers (amounts) on the left to right oriented number line might be linear. In a previous work (Cammaerts \& Cammaerts, 2019f), we assessed the ants' responses to the subtractions 2 minus 1, 3-1, 4-1 and 5-1, and obtained the mean scores of $94.0 \%, 90.2 \%, 85.3 \%$, and $82.2 \%$ respectively. For having an idea of the relation between the level of the ants' response to the sighted amounts and these amounts, we arbitrarily replaced 2-1, 3-1, 4-1, 5-1, by the amounts approaching the best what they saw, i.e. $1.5,2.5,3.5,4.5$. The graph shows that the ants reacted less and less well for larger and larger numbers and that their responses seem to linearly decrease with these amounts (empty circles). Their response seems to less well linearly decrease with the decimal logarithm of these amounts $(0.17,0.39,0.54$, and 0.65$)$ (black squares). The values of the amounts and of their decimal logarithms are reported on the $\mathrm{x}$ axis; the ants' responses are reported on the y axis.

\section{Discussion - Conclusion}

Using six ant colonies and making three different experiments, we found that these insects mentally situated the smaller amounts of elements on the left of larger ones, and the larger ones on the right of smaller ones, this including the zero element. This result is in agreement with the finding that the workers of the ant $M$. sabuleti locate the zero at the start of an increasing series of elements and at the end of a decreasing series of elements (Cammaerts \& Cammaerts, 2019e). We here can add that, for the ants, the zero is the 'most to the left' value.

The unexpected finding of a number arrangement on a left to right oriented scale in ants corresponds to the ordering scale for numbers or amounts that exists in humans and in a few already studies vertebrates (see the introduction section). These individuals possess what has been labeled a 'number line', on which the smaller values are situated on the left and the larger ones on the right (Dehaene, 2011). This 'number line' seems to be natively detained, and 
in humans, is independent of the kind of writing specific to civilizations and education (Rugani \& de Hevia, 2017). In their number representation, humans perceive the difference between two consecutive values as becoming smaller as the values become larger, even if such a difference has an identical absolute value (e.g. the difference between 1 and 2 and the difference between 1,001 and 1,002). They thus arrange the numbers on their number line according to a logarithmic function, with natively the smaller values on the left and the larger ones on the right (Dehaene et al., 1998; Rugani \& de Hevia, 2017; Vallortigara, 2018: at the end of his paper; Dehaene, 2011, p. $65)$.

Although not specifically based on the results of the present experimental work, the kind of arrangement of numbers in the left to right oriented number line of the M. sabuleti ants could be deduced from the results of a previous work on the subtracting abilities of this ant species (Cammaerts \& Cammaerts, 2019f). These ants' responses to the scaling of numerosities appear to correspond better to a linear function than to a logarithmic function, but further investigations are necessary before concluding.

In a part of his recent review about the origin of numerosity abilities, Vallortigara (2018) advances that a nativistic foundation of number-space association seems to exist in humans and animals, and tries to find an explanation. However, he states that similar behavior may results from different structures and mechanisms functionally convergent.

In humans and vertebrates, the numerosity ability is processed by specific neurons of particular brain zones (Verguts \& Fias, 2006). Such studies of the cerebral activity under mathematical stimulation might be undertaken on ants, in the manner they have been done for olfactory stimulation (Lopez-Riquelme, Hermandez-Falcon, \& Ramon, 2009).

In conclusion and unexpectedly, M. sabuleti ants appeared to arrange numbers (amounts) on a scale locating the smaller ones on the left and the larger ones on the right, as do humans and some vertebrates already studied as for this characteristic. Moreover, this is in agreement with the ants' locating the zero at its due mathematical place. The ants' discrimination between two successive numbers (or amounts) decreases as the numbers become larger. Their arrangement of numbers on a left to right scale might be linear but further investigations are required to define this topic.

\section{Conflict of interests}

The authors declare that there is no conflict of interests regarding the publication of this paper.

\section{References}

Agrillo, C. (2012). Evidence for two numerical systems that are similar in humans and guppies. PLoS ONE, 7(2), e31923.

Brannon, E. M., \& Herbert, T. S. (1988). Ordering of the numerosities 1 to 9 by monkeys. Science, New Series, 282(5389), 746-749.

Brannon, E. M., Wusthoff, C. J., Gallistel, C. R., \& Gibbon, J. (2001). Numerical subtraction in the pigeon: Evidence for a linear subjective number scale. Psychological Science, 12(3), 238-243. Retrieved from https://psycnet.apa.org/record/2001-01364-009

Cammaerts, M.-C. (2007). Colour vision in the ant Myrmica sabuleti MEINERT, 1861 (Hymenoptera: Formicidae). Myrmecological News, 10, 41-50.

Cammaerts, M.-C. (2008). Visual discrimination of cues differing as for their number of elements, their shape or their orientation, by the ant Myrmica sabuleti. Biologia, 63, 1169-1180. Retrieved from https://link.springer.com/article/10.2478/s11756-008-0172-2

Cammaerts, M.-C., \& Cammaerts, D. (2009). Light thresholds for colour vision in the workers of the ant Myrmica sabuleti (Hymenoptera: Formicidae). Belgian Journal of Zoology, 138, 40-49.

Cammaerts, M.-C., \& Cammaerts, R. (2019a). Ants are at the first stage of the notion of zero. International Journal of Biology, 11(1), 54-65.

Cammaerts, M.-C., \& Cammaerts, R. (2019b). Ants correctly locate the zero in a continuous series of numbers. International Journal of Biology, 11(4), 25-34.

Cammaerts, M.-C., \& Cammaerts, R. (2019c). Ants fail to add numbers of same elements seen consecutively. International Journal of Biology, 11(3), 37-48.

Cammaerts, M.-C., \& Cammaerts, R. (2019d). Ants' capability of adding numbers of identical elements. International Journal of Biology, 11(3), 25-36. 
Cammaerts, M.-C., \& Cammaerts, R. (2019e). Ants' notion of zero through the perception of the absence of an odor. International Journal of Biology, 11(2), 1-12.

Cammaerts, M.-C., \& Cammaerts, R. (2019f). Subtraction-like effect in an ant faced with numbers of elements which includes a crossed one. International Journal of Biology, 11(4), 51-66.

Dehaene, S. (2011). The Number Sense. New York, Oxford, University Press. Retrieved from https://psycnet.apa.org/record/2011-10610-000

Dehaene, S., Dehaene-Lamberts, G., \& Cohen, L. (1998). Abstract representations of numbers in the animal and human brain. Trends in Neurosciences, 21(8), 355-61. Retrieved from http://www.unicog.org/../Dehaene _ReviewNumbersAnimalsInfants_TIN...

Hauser, M. D., Carey, S., \& Hauser, L. B. (2000). Spontaneous number representation in semi-free-ranging rhesus monkeys. Proceedings of the Royal Society of London B: Biological Sciences, 267(1445), 829-833.

Lopez-Riquelme, G. O., Hermandez-Falcon, J., \& Ramon, F. (2009). Correlations between antennae (electroantennogram) and brain (ERPs) response during odour stimulation in ants. Program N0 68.20/V4. Neuroscience Meeting Planner. Chicago, IL: Society for Neuroscience. Online. Retrieved from https://www.researchgate.net/...Antennae_Electroantennogram...Br...

Rugani, R., \& de Hevia, M.-D. (2017). Number-space associations without language: Evidence from preverbal human infants and non-human animal species. Psychonomic Bulletin \& Review, 24(2), 352-369. Retrieved from https://link.springer.com/article/10.3758/s13423-016-1126-2

Rugani, R., Vallortigara, G., Priftis, K., \& Regolin, L. (2015). Number-space mapping in the newborn chick resembles humans' mental number line. Science, 347(6221), 534-536.

Siegel, S., \& Castellan, N. J. (1989). Nonparametric statistics for the behavioural sciences. Singapore, McGrawHill Book Company. Retrieved from https://www.amazon.com/Sidney-Siegel... Statistics.../B008WDIR6

Vallotigara, G. (2018). Comparative cognition of number and space: The case of geometry and of the mental number line. Philosophical Transactions of the Royal Society B: Biological Sciences, 373.

Verguts, T., \& Fias, W. (2004). Representation of number in animals and humans: A neural model. Journal of Cognitive Neuroscience, 16(9), 1493-1504.

\section{Copyrights}

Copyright for this article is retained by the author(s), with first publication rights granted to the journal.

This is an open-access article distributed under the terms and conditions of the Creative Commons Attribution license (http://creativecommons.org/licenses/by/4.0/). 